\title{
Analysis of Repairable $k$-out-of- $n$ System Model Using Inter-Arrival Failure Times
}

\author{
Mehmet GÜRCAN ${ }^{1}$, Yunus GÜRAL ${ }^{1}$, and Gökhan GÖKDERE ${ }^{1, *}$ \\ ${ }^{1}$ Department of Statistics, University of Firat, Elazı $\breve{g}$, Turkey
}

\begin{abstract}
Examination of repairable $k$-out-of- $n$ system which has an important place in systems is an important application area of reliability theory. In general, the reliability analysis of a repairable system is based on the distribution of the lifetimes of the components in the system. In this paper, the reliability analysis of the repairable $k$-out-of- $n$ system is made through the inter-arrival failure times of the components in the system.
\end{abstract}

\section{Introduction}

A working system is defined as a dynamic structure. When the system is observed the time of observation forms the section of the working system. In systems with dynamic structure, the most important problem is the moment when the system should be examined. This problem can be solved with the help of stochastic processes with continuous parameters. In general, a dynamic system is observed at a time when it changes its states. Since the system which is examined is a repairable system, it needs to be considered at failure times of the components.

As the system is examined at failure times of the components, the random variable that is important to us is the number of failed components in the repair. Let $X(t)$ denotes the number of failed components in the repair at time $t, t \geq 0$. Note that, the distribution of the time between successive failures of the components may be different from the exponential distribution. Therefore, $X(t)$ is not a Markov or semi-Markov process. However, a semiMarkov process can be obtained with the help of the $X(t)$, which represents the times of failure of the system. Let $t_{k}$ represents the moment that the $k$-th failure takes place. At the end of the $t_{k}$, let $X\left(t_{k}-0\right)=X_{k}$ denotes the number of failed components in the repair, excluding the last failed component. Then, the process $\{\xi(t), t \geq 0\}$ with $\xi(t)=X_{k}, t_{k} \leq$ $t<t_{k+1}$ becomes a semi-Markov process. The state space of this process becomes $\{0,1,2, \ldots, k\}$ in the repairable $k$-out-of- $n$ system. Also, $t_{1}^{k}, t_{2}^{k}, \ldots$ are the times of arrival to the case of $k$.

\footnotetext{
*Corresponding author: g.g.gokdere@gmail.com
} 
Thus, in the process $\xi(t)$, the first failure time of the repairable $k$-out-of- $n$ system and the elapsed time between successive failure times are reduced to the finding the distributions of the first arrival time and return time to the case of $k$. Note that, after the first failure of the system, it is being repaired and starting to work.

Assume that the repair time in the system is random variable with exponential distribution with parameter $\mu$. Let, $F$ be the cumulative distribution function of the times between successive failure times of the components. The function $a_{r}(s), r=0,1, \ldots s \geq 0$ is defined by

$$
a_{r}(s)=\int_{0}^{\infty} \frac{(\mu t)^{r}}{r !} e^{-(s+\mu) t} d F(t)
$$

Let $f(s)$ be the Laplace transformation of $F(x)$. Determination $d_{k}$ is defined as

$$
d_{k}=\operatorname{det}\left[\begin{array}{cccccc}
1 & -a_{0} & & & & \\
1 & 1-a_{1} & -a_{0} & & & \\
\vdots & \vdots & \vdots & & & \\
1 & -a_{k-2} & -a_{k-3} & \cdots & 1-a_{1} & -a_{0} \\
1 & -a_{k-1} & -a_{k-2} & \cdots & -a_{2} & 1-a_{1}
\end{array}\right]
$$

where

$$
a_{r}=a_{r}(0)=\int_{0}^{\infty} \frac{(\mu t)^{r}}{r !} e^{-\mu t} d F(t) .
$$

Let $T_{0 k}$ be the first failure time of the system and $T_{k k}$ be the time between successive failures. Then we have

$$
\begin{gathered}
E T_{0 k}=\frac{d_{k}}{\alpha^{k}} m \\
E T_{k k}=\left(\frac{d_{k}}{\alpha^{k}}-\frac{d_{k-1}}{\alpha^{k-1}}\right) m,
\end{gathered}
$$

where

$$
m=-f^{\prime}(0)
$$

and

$$
\alpha=f(\mu)=\int_{0}^{\infty} e^{-\mu t} d F(t)
$$

Here, $m$ is the average time between failures of the components and $\alpha$ is the probability that the repair time is greatest among the failures. 


\section{Illustrative Example}

As an illustration, consider a repairable 10-out-of- $n$ system. Assume that the distribution of components is exponential distribution with the parameter $\mu=0.025$. The distribution of the times between successive failures of the system is given in Table 1.

Table 1. The distribution of the times between successive failures of the system

\begin{tabular}{ccl}
\hline No & $t_{k+1}-t_{k}$ & $F(t)$ \\
\hline 1 & 12 & 0.01 \\
2 & 20 & 0.085 \\
3 & 35 & 0.21 \\
4 & 45 & 0.27 \\
5 & 50 & 0.42 \\
6 & 55 & 0.57 \\
7 & 60 & 0.78 \\
8 & 65 & 0.905 \\
9 & 72 & 1 \\
\hline
\end{tabular}

From the above table we can get the following results:

1. The mean of the times between successive failures is $m=50.21$.

2. $d_{10}=0.0188, d_{9}=0.0358$ and $\alpha=0.2876$.

3. The mean time to first failure of the system is $E T_{0 k}=2.4 \times 10^{5}$.

4. The mean time between successive failures is $E T_{k k}=1.1 \times 10^{5}$.

\section{Conclusions}

Studies related to the reliability of the systems are carried out using the distribution of the life time of the components. In this paper, we have studied repairable $k$-out-of- $n$ system under the assumption that elapsed time between components is known. We have obtained two important results. In the first of the results, the mean time until the moment of failure can be calculated. In the second result, it is possible to calculate the mean running time between successive failures. These results are very important for preventive maintenance of the repairable $k$-out-of- $n$ system. Also, the number of the components in the system has no importance since the distribution of the lifetime of the components are not consideration in the examination. 


\section{References}

1. F.S.Q. Alves, H.C. Yehia, L.A.C. Pedrosa, F.R.B. Cruz and L. Kerbache, Upper bounds on performance measures of heterogeneous $\mathrm{M} / \mathrm{M} / \mathrm{c}$ queues, Mathematical Problems in Engineering, Article ID 702834 (2011).

2. J.B. Atkinson, Two new heuristics for the GI/G/n/0 queueing loss system with examples based on the two-phase Coxian distribution, Journal of the Operational Research Society, 60, 818-830 (2009).

3. S.L. Brumelle, A generalization of Erlang's loss system to state dependent arrival and service rates, Mathematics of Operations Research, 3, 10-16 (1978).

4. E. Çinlar, Markov renewal theory, Advances in Applied Probability, 12, 123-187 (1969).

5. S. Halfin, Distribution of the interoverflow time for the GI/G/1 loss system, Mathematics of Operations Research, 6, 563-570 (1981).

6. H.O. Isguder and C.C. Celikoglu, Minimizing the loss probability in GI/M/3/0 queueing system with ordered entry, Scientific Research and Essays, 7, 963-968 (2012).

7. R. Pyke, Markov renewal processes: Definitions and preliminary properties, Annals of Mathematical Statistics, 32, 1231-1242 (1961a).

8. R. Pyke, Markov renewal processes with finitely many states, Annals of Mathematical Statistics, 32, 1243-1259 (1961b).

9. W.L. Smith, Regenerative stochastic processes, Proceedings of the Royal Society of London, Series A, Mathematical and Physical Sciences, 232, 6-31 (1955). 